\title{
New Characterizations for the Timelike Curve by the help of Spherical Representations in Minkowski 3-Space
}

Mihriban KÜLAHCI ${ }^{1}$

ABSTRACT: In this paper, some new characterizations have been obtained by using arc length and harmonic curvature function of spherical represantations for the timelike curve in Minkowski 3-space.

Keywords. Frenet frame, helix, minkowski 3-space.

\section{Minkowski 3-Uzayında Küresel Temsiller Yardımıyla Timelike Ĕgri İçin Yeni Karakterizasyonlar}

ÖZET: Bu makalede, Minkowski 3-uzayında bir timelike eğrisi için küresel temsillerinin yay uzunluğunu ve harł monik eğrilik fonksiyonunu kullanarak bazı yeni karakterizasyonlar elde edildi.

Anahtar Kelimeler. Frenet çatısı, helis, minkowski 3-uzayı.

Fırat Üniversity, Science Faculty, Mathematic Department, Elazığ, Turkey

Sorumlu yazar/Corresponding Author: Mihriban KÜLAHCI, mihribankulahci@gmail.com 


\section{INTRODUCTION}

Let $I R_{1}^{3}$ denote the 3-dimensional Lorentz space, i.e. the usual vector space $I R_{1}^{3}$ with the Lorentz scalar product of $x$ and $y$ is given by

$\langle x, y\rangle=-x_{1} y_{1}+x_{2} y_{2}+x_{3} y_{3}$

where $x=\left(x_{1}, x_{2}, x_{3}\right)$ and $y=\left(y_{1}, y_{2}, y_{3}\right)$ are two vectors in $I R^{3}$.

$I R_{1}^{3}$ is called three-dimensional Lorentz space or Minkowski 3-space. We denote $L^{3}$ as $I R_{1}^{3}$.

Recall that a vector $x$ in $L^{3}$ can have one of three casual characters: it can be spacelike if $\langle x, x\rangle>0$, timelike $\langle x, x\rangle<0$, and null. $\langle x, x\rangle=0, x \neq 0$. For $x \in L^{3}$, the norm of a vector $x$ is given by $\|x\|=\sqrt{|\langle x, x\rangle|}$, and $x$ is called a unit vector if $\|x\|=1$.

Similarly, an arbitrary curve $\alpha(t)$ can be locally spacelike, timelike or null (lightlike), if all of its velocity vectors $\alpha^{\prime}(t)$ are spacelike, timelike or null (lightlike), respectively. (O'neill, 1983).

Let's give the definition of Darboux vector. Vectors $t, n, b$ change while a point $P$ on the curve drawing the curve. Hence these vectors constitute of spherical images of curve. Suppose that Frenet vectors $\{t, n$, $b$ of the curve makes an abrupt helix motion about an axis at each $s$ time. This axis is called Darboux axis corresponding $s$ parameter at $\alpha(s)$ point. The vector obtained oriented and direction of this axis is called Darboux vector at point $\alpha(s)$ of the curve (Yücesan et al., 2004).

In differential geometry of curves in Euclidean space and Lorentzian space, helix is a well-known concept. Harmonic curvatures have an important role in the characterizations of helices. Many studies on harmonic curvatures and helices have been done by many mathematicians (Sakomato, 1982; Barros, 1997; Arslan et al.;2000; Ekmekçi, 2000; İyigün and Arslan, 2005; Külahc1 et al.,2009). Furthermore, in recent years, many important and intensive studies have been seen about inclined curves (Hacisalihoğlu, 2009; Ghadami, 2012).

The aim of this paper is to implement the results which were given in (Öğrenmiş et al., 2014) to arc lengths of spherical representations of T, N, B for a timelike space curve in Minkowski 3-space. Furthermore, by considering Darboux vector as given in (Yücesan et al., 2004), we give the arc lengths of spherical representations of the vector field $\vec{C}=\frac{\vec{w}}{\| \vec{w} \mid}$.

\section{MATERIAL AND METHODS}

Let $\{t, n, b\}$ be the Frenet vectors of the differentiable timelike space curve in Minkowski space. Then the Frenet equations are

$$
\begin{aligned}
t^{\prime} & =\kappa n, \\
n^{\prime} & =\kappa t+\tau b, \\
b^{\prime} & =-\tau n,
\end{aligned}
$$

where $\kappa$ and are curvature and torsion, respectively (Yücesan et al., 2004).

In addition, Darboux vector can be given as follows (Yücesan et al., 2004):

$\vec{w}=-\tau \vec{t}-\kappa \vec{b}$

Definition 2.1. In n-dimensional Lorentzian space, $H_{i}: I \rightarrow R$ function for a time-like curve is defined as follows:

$H_{i}(s)=\left\{\begin{array}{cl}0 & , i=0 \\ \frac{\kappa_{1}}{\kappa_{2}} & , i=1 \\ \left\{V_{1}\left[H_{i-1}\right]+\varepsilon_{0} H_{i-2} k_{i}\right\} \varepsilon_{0} & , 1<i \leq n-2\end{array}\right.$

is called $\mathrm{i}^{\text {th }}$ order harmonic curvature function of the curve.

$\varepsilon_{1}=\left\{\begin{array}{cc}-1 & , V_{i} \text { time }- \text { like } \\ 1 & , V_{i} \text { space }- \text { like }\end{array}\right.$

where $V_{1}$ is unit tangent vector field and $\kappa_{1}, \kappa_{2}, \ldots, \kappa_{n-1}\left(\kappa_{n-1} \neq 0\right)$ is a curvature function of the curve (Soylu, et al., 1999).

\section{RESULTS AND DISCUSSION}

Theorem 3.1. $\alpha \subset L^{3}$ is an ordinary helix if and only if

$s_{t}=\tau H s+c$. 
Proof. Let $t=t(s)$ be the tangent vector field of the curve

$$
\begin{aligned}
\alpha: I \subset R & \rightarrow L^{3} \\
s & \rightarrow \alpha(s)
\end{aligned}
$$

The spherical curve $\alpha_{t}=t$ on $S^{2}$ is called first spherical representation of the tangent of $\alpha$.

Let $s$ be the arc length parameter of $a$. If we indicate the arc length of the curve $\alpha_{t}$ by $s$ then one can write $\alpha_{t}(s)=t(s)$

Letting $\frac{d a_{t}}{d s_{t}}=t_{t}$, we have $t_{t}=\kappa \vec{n} \frac{d s}{d s_{t}}$. Hence one can get $\frac{d s_{t}}{d s}=\kappa$. Thus we give the following result.

If $\kappa$ is the first curvature of the curve $\alpha: I \rightarrow L^{3}$, then the arc length $S_{1}$ of the tangentian representation $\alpha_{t}$ of $\alpha$ is

$s_{t}=\int \kappa d s+c$.

If the harmonic curvature of $\alpha$ is $H=\frac{\kappa}{t}$, one can have

$$
s_{t}=\int \tau H d s+c
$$

where $c$ is an integral constant.

Theorem 3.2. $\alpha \subset L^{3}$ is an ordinary helix if and only if

$$
S_{n}=\tau \sqrt{1+H^{2}} s+c .
$$

Proof. Let $\vec{n}=\vec{n}(s)$ be the principal normal vector field of the curve

$$
\begin{aligned}
\alpha: I \subset R & \rightarrow L^{3} \\
s & \rightarrow \alpha(s)
\end{aligned}
$$

The spherical curve $\alpha_{n}=\vec{n} \quad$ on $\quad S^{2}$ is called second spherical representation for $\alpha$ or is called the spherical representation of the principal normals of $\alpha$ Let $s \in I$ be the arc length parameter of $\alpha$. If we denote the arc length of the curve $\alpha$. by $S_{n}$, one can write $\alpha_{n}\left(s_{n}\right)=\vec{n}(s)$.

Furthermore letting $\frac{d a_{n}}{d s_{n}}=T_{n}$, one can obtain

$$
T_{n}=(\kappa \vec{t}+\tau \vec{b}) \frac{d s}{d s_{n}} .
$$

Thus, one can have

$$
\frac{d s_{n}}{d s}=\sqrt{\kappa^{2}+\tau^{2}} .
$$

Note that $\sqrt{\kappa^{2}+\tau^{2}}$ is the total curvature function of $\alpha$. Moreover one can get the following result:

$s_{n}=\int \sqrt{\kappa^{2}+\tau^{2}} d s+c$

or in terms of $H=\frac{k}{t}$,

$s_{n}=\int t \sqrt{1+H^{2}} d s+c$.

Theorem 3.3. $\alpha \subset L^{3}$ is an ordinary helix if and only if

$$
s_{b}=\frac{\kappa}{H} s+c .
$$

Proof. Let $\vec{b}=\vec{b}(s)$ be the binormal vector field of the curve

$$
\begin{aligned}
\alpha: I \subset R & \rightarrow L^{3} \\
s & \rightarrow \alpha(s)
\end{aligned}
$$

The spherical curve $\alpha_{b}=\vec{b}$ on $S^{2}$ is called third spherical representation for $\alpha$ or is called the spherical representation of the binormal of $\alpha$.

Let $s \in I$ be the arc length parameter of $\alpha$. If we 
denote the arc length parameter of the curve $\alpha_{b}$ by $s_{b}$, one can write

$$
\alpha_{b}\left(s_{b}\right)=\vec{b}(s) \text {. }
$$

Moreover letting $\frac{d \alpha_{b}}{d s_{b}}=t_{b}$, one can obtain

$$
t_{b}=-\tau \vec{n} \frac{d s}{d s_{b}}
$$

Hence one can have $\frac{d s_{b}}{d s}=\tau$ and $s_{b}=\int \tau d s+c$ or in terms of the harmonic curvature of $\alpha$ one can get

$$
s_{b}=\int \frac{k}{H} d s+c .
$$

Theorem 3.4. The curve $\alpha \subset L^{3}$ is an ordinary helix if and only if

$$
s_{c}=\int \frac{H^{\prime}}{H^{2}} d s+c .
$$

Proof. $\alpha \subset L^{3}$. Let $\vec{w}=-t \vec{t}-k \vec{b}$ be the Darboux vector field of the curve

$$
\begin{aligned}
\alpha: I \subset R & \rightarrow L^{3} \\
s & \rightarrow \alpha(s) .
\end{aligned}
$$

Let us define the curve $a_{c}=\vec{c}$ on $S^{2}$ by the help of the vector field $\vec{c}=\frac{\vec{w}}{\|\vec{w}\|}$. This curve is called IV. th spherical representation of $\alpha$ or is called the Darboux representation of $\alpha$. Let $s_{c}$ be the arc length of $\alpha_{c}$. Then one can have $\alpha_{c}=\vec{c}\left(s_{c}\right)=\frac{\vec{w}}{\| \vec{w} \mid}$. Let us denote the hyperbolic angle between $\vec{w}$ and $\vec{t}$ by $\varphi$.

\section{Hence}

$\kappa=\|\vec{w}\| \sinh \varphi \quad$ and $\tau=\|\vec{w}\| \cosh \varphi$.
Therefore, one can write

$$
\vec{c}=\cosh \varphi \vec{t}+\sinh \varphi \vec{b} .
$$

From this last equality one can obtain

$$
\frac{d \vec{c}}{d s_{c}}=\frac{d \vec{c}}{d s} \cdot \frac{d s}{d s_{c}}
$$

or

$$
\frac{d s_{c}}{d s}=\left|\frac{d \vec{c}}{d s}\right|
$$

$$
\text { or }
$$

$$
\begin{aligned}
\frac{d \vec{c}}{d s} & =(\cosh \varphi)^{\prime} \vec{t}+(\sinh \varphi)^{\prime} \vec{b} \\
& =(\sinh \varphi \vec{t}+\cosh \varphi \vec{b}) \frac{d \varphi}{d s} .
\end{aligned}
$$

Hence one can have

$$
\left\|\frac{d \vec{c}}{d s}\right\|=\frac{d j}{d s}=\frac{d s_{c}}{d s} .
$$

Considering these equations and (11), one can obtain

$$
\frac{\kappa}{\tau}=\tanh \varphi .
$$
have

Therefore, differentiating with respect to $s$, one can

$$
\left(\frac{\kappa}{\tau}\right)^{\prime}=\frac{1}{\operatorname{coth}^{2} \varphi} \frac{d \varphi}{d s}
$$

$$
\left(\frac{\kappa}{\tau}\right)^{\prime}=\left[\frac{1}{\left(\frac{\tau}{\kappa}\right)^{2}}\right] \frac{d \varphi}{d s} .
$$

From (17), one can get

$$
\frac{d \varphi}{d s}=\frac{\left(\frac{\kappa}{\tau}\right)^{\prime}}{\left(\frac{1}{\tau}\right)^{2}}
$$


and since $H=\frac{\kappa}{\tau}$, one can obtain

$\frac{d \varphi}{d s}=\frac{H^{\prime}}{H^{2}}$

Hence from (16), one can have

$\frac{d s_{c}}{d s}=\frac{H^{\prime}}{H^{2}}$

or hence

$$
d s_{c}=\frac{H^{\prime}}{H^{2}} d s \text {, }
$$

the equation(23) implies that

$s_{c}=\int \frac{H^{\prime}}{H^{2}} d s+c$.

\section{REFERENCES}

Arslan K, Çelik Y, and Hacısalihoğlu HH, 2000. On Harmonic Curvatures Of A Frenet Curve. Commun. Fac. Sci. Univ. Ank. Series A1, 49: 15-23.

Barros M, 1997. General helices and a theorem of Lancert. Proc. AMS, 125:1503-1509.

Ekmekçi N, Hacısalihoğlu HH, and İlarslan K, 2000. Harmonic curvatures in Lorentzian space. Bull. Malaysian Math. Sc. Soc. (Second Series), 23: 173-179.

Ghadami R, Yayl1 Y, 2012. A new characterization for inclined curves by the help of spherical representations according to Bishop frame. International Journal of Pure and Applied Mathematics, 74(4):455-463.

Hacısalihoğlu HH, 2009. A New Characterization For Inclined Curves By The Help Of Spherical Representations. International Electronic Journal Of Geometry, 2(2): 71-75.

İyigün E, and Arslan K, 2005. On Harmonic Curvatures of Curves in Lorentzian N-Space. Commun. Fac. Sci. Univ. Ank. Series A1, 54(1):29-34.

Külahcı M, Bektaş M, and Ergüt M, 2009. On Harmonic Curvatures of A Frenet Curve in Lorentzian Space. Chaos, Solitons and Fractals 41: 1668--1675.

O’Neill B, 1983. Semi-Riemannian Geometry with Applications to Relativity. Academic Press Inc., London, England, 468 p.

ÖğrenmişAO, Yılmaz MY, Külahcı M, 2014. Some characterizations for curves by the help of spherical representations in the Galilean and pseudo-Galilean space. Acta Universitatis Apulensis, 40:373-380.

Sakomato, K, 1982. Helical immersions into a unit sphere. Math. Ann. 261:63-80.

Soylu D, Bektaş M, Ergüt M, 1999. Characterizations for osculator hypersphere in Lorentzian space. Jour of Inst of Math \& Comp Sci (Math Ser), 12(3): 229-35.

Yücesan A, Çöken AC, Ayyıldız N, 2004. On the darboux rotation axis of Lorentz space curve. Applied Mathematics and Computation, 155: 345-351. 
